\title{
Reviews
}

Pharmacology

\section{Drug-Induced Pseudoallergy: A Review of the Causes and Mechanisms}

\author{
Bo Zhang Qin Li Chenyang Shi Xinyue Zhang \\ Department of Pharmacology, Institute of Materia Medica, Zhejiang Academy of Medical Sciences, \\ Hangzhou, PR China
}

\section{Keywords}

Complement · Drugs · Mast cells · Pseudoallergy

\begin{abstract}
Adverse drug reactions occur frequently and can trigger pseudoallergy, which has become a serious threat to public health. Pseudoallergy is a typical non-immune anaphylactic reaction characterized by the independence of antigenspecific immune responses. In the clinic, pseudoallergy is often elicited by the first dose of medication, and here lies its unpredictability and occasional lethal outcome. However, the mechanisms of pseudoallergy are not well understood. This review focusses on the causes and mechanisms of pseudoallergy induced by drugs. Two categories of mechanisms will be considered, namely, (1) complement activation-related pseudoallergy and (2) mast cell activation-related pseudoallergy. The factors that induce pseudoallergy include opioid drugs, complement activation-related pseudoallergenic drugs, nonsteroidal anti-inflammatory drugs and traditional Chinese medicine injections.
\end{abstract}

(c) 2017 S. Karger AG, Basel

\section{Introduction}

Hypersensitivity reactions (HSRs) have been categorized into 4 groups [1]. However, the classification of HSRs by Gell and Coombs has limitations in that pseudo- allergy cannot be fitted into any of the 4 types $[1,2]$. Pseudoallergy is characterized by immediate systemic reactions that are similar to anaphylaxis symptoms, but the mechanisms involved are mediated by the release of mediators from basophils and mast cells that is not triggered by Immunoglobulin E (IgE) [3]. The condition is often elicited by the first dose of medication, which induces mast cells and basophils to degranulate. Pseudoallergy does not elicit antigen-specific immune responses but does evoke histamine release, the activation of the complement system, atypical synthesis of eicosanoids, and the inhibition of bradykinin breakdown [4].

Indeed, pseudoallergy and anaphylaxis are clinically indistinguishable [5]. The signs and symptoms of pseudoallergy are practically identical to IgE-mediated symptoms including angioedema, urticaria, bronchospasm and gastrointestinal signs $[6,7]$, together with skin flushing, headache, edema, hypotension and shock [8]. Pseudoallergy can be attributed to the direct activation of effector cells and the form of anaphylatoxins produced and may be induced by opioid drugs, complement activationrelated pseudoallergenic drugs, nonsteroidal anti-inflammatory drugs (NSAIDs) and traditional Chinese medicine injections (TCMIs).

It has been suggested that two-thirds of HSRs may be attributable to pseudoallergy [9]. Due to the lack of systematic studies of the causes and mechanisms involved in pseudoallergy, the diagnosis and treatment of these reactions remain a significant clinical challenge. Therefore,

\section{KARGER}

(c) 2017 S. Karger AG, Basel

E-Mail karger@karger.com

www.karger.com/pha
Dr. Xinyue Zhang

Department of Pharmacology, Institute of Materia Medica

Zhejiang Academy of Medical Sciences

Hangzhou, Zhejiang 310013 (PR China)

E-Mail zhangxy2003@aliyun.com 
this review clarifies some of the possible causes and mechanisms involved in drug-induced pseudoallergy to facilitate clear thinking.

\section{Nomenclature}

As early as 1937, the term "anaphylactoid reaction" was used to describe a syndrome caused by the injection of egg white. In 1950, Halpern and Briot described this syndrome in great detail. In 1957, Parratt and West [10] suggested that the anaphylactoid reaction was noted mainly in the rat. However, Ehlers et al. [11] identified the roles of non-allergic HSRs in young people with chronic urticaria. In the 1990s, the terms "pseudoallergy" and "non-allergic hypersensitivity" began to be used frequently. Various authors reported the HSRs as pseudoallergen-induced. The prefix "pseudo-" is commonly used to describe the symptoms that mimic another condition. The clinical symptoms of pseudoallergy have been shown to be similar to those of classical anaphylaxis [12]. It is important that medical terminology is widely understood by all specialists and physicians engaged in different fields of medicine and research. In view of inconsistency in the terminology in the published literature, we believe that "pseudoallergy" should be the preferred term for this condition.

\section{Mechanisms of Pseudoallergy}

Although the clinical symptoms of pseudoallergy are quite similar to common allergy or type 1 reactions [12], pseudoallergy is quite distinct from IgE-mediated allergy. The reaction arises for the first time without prior sensitization [13] and different drugs can trigger pseudoallergy by different mechanisms, that is, taxol can induce pseudoallergy by activating the complement system [13].

\section{Complement Activation-Related Pseudoallergy}

Anaphylatoxins are the causative factors that trigger complement activation-related pseudoallergy (CARPA). Complement-mediated reactions counter the effects of potentially harmful agents in the body (radiocontrast media, liposomes, NSAIDs, TCMIs) through the anaphylatoxin/mast cell/circulatory system axis $[1,14]$. The centre of CARPA is the complement system, which causes subsequent reactions in the CAPRA cascade. Molecular and cellular activation and interactions comprise this complicated biochemical cascade. In principle, the processes un- derlying CARPA consist of 3 steps: (1) the complement system is activated; (2) secretory cells and blood cells are stimulated; and (3) effector cells of CARPA respond to mediator challenges [14]. The initial trigger activates the complement system, which induces the release of the primary mediators. Then, the anaphylatoxins bind to secretory cells (mast cells, basophils, macrophages, other phagocytic cells, and leukocytes) leading to the release/ production of a multitude of secondary mediators (including histamine, tryptase, platelet-activating factor, thromboxane $\mathrm{A}_{2}$, leukotrienes (LTs), cytokines, proteases, and prostaglandins) $[14,15]$. The complement cascade can be triggered by the classical pathway (CP), lectin pathway (LP) or alternative pathway (AP) [16] in which complement component $3(\mathrm{C} 3)$ plays an important role (Fig. 1). C3, a protein associated with complement cascades, is critical to the complement system [17]. The membrane attack complex is formed by the activity of these 3 pathways.

\section{Classical Pathway}

The CP is triggered by interactions between antigenantibody complexes on the surface of microorganisms [18]. The proteases $\mathrm{C} 1 \mathrm{~s}$ are part of the $\mathrm{C} 1$ complex. $\mathrm{C} 1 \mathrm{~s}$ can mediate the generation of $\mathrm{CP} \mathrm{C} 3$ convertase. $\mathrm{Clq}$ binds to Immunoglobulin $\mathrm{G}$ (IgG) and Immunoglobulin $\mathrm{M}$ promoting cascade progression, which causes $\mathrm{C} 1 \mathrm{r}$ and $\mathrm{C} 1$ s activation. $\mathrm{C} 4$ is cleaved into $\mathrm{C} 4 \mathrm{~b}$ by this protease. Following cleavage of $\mathrm{C} 2, \mathrm{C} 4 \mathrm{~b} 2 \mathrm{a}$ is generated, which converts $\mathrm{C} 3$ into $\mathrm{C} 3 \mathrm{~b}$ and forms $\mathrm{C} 4 \mathrm{bC} 2 \mathrm{aC} 3 \mathrm{~b}$ (C5 convertase) [19-21]. The C3 convertase C4b2a is able to cleave C 3 and initiate the amplification of downstream effector functions $[22,23]$.

\section{Lectin Pathway}

The LP involves the activity of mannose-binding lectin (MBL) and ficolins acted pattern-recognition molecule that directly recognizes carbohydrate patterns on the surface of pathogenic microorganism. Each patternrecognition molecule that assembles with MBL-associated serine proteases (MASPs) has a similar structure to $\mathrm{C} 1 \mathrm{r}$ or $\mathrm{C} 1 \mathrm{~s}$. On this basis, MASP-2 cleaves $\mathrm{C} 4$ and $\mathrm{C} 2$, which induce generation of the $\mathrm{C} 3$ convertase [22].

\section{Alternative Pathway}

The AP of the complement system is an innate component of the immune system, playing an important role in the natural defense against infection. AP is different from CP and LP; AP C3 can be directly activated and can 
Fig. 1. Classical, lectin and alternative pathways. MBL, mannose-binding lectin; MASPs, MBL-associated serine proteases; MAC, membrane attack complex.

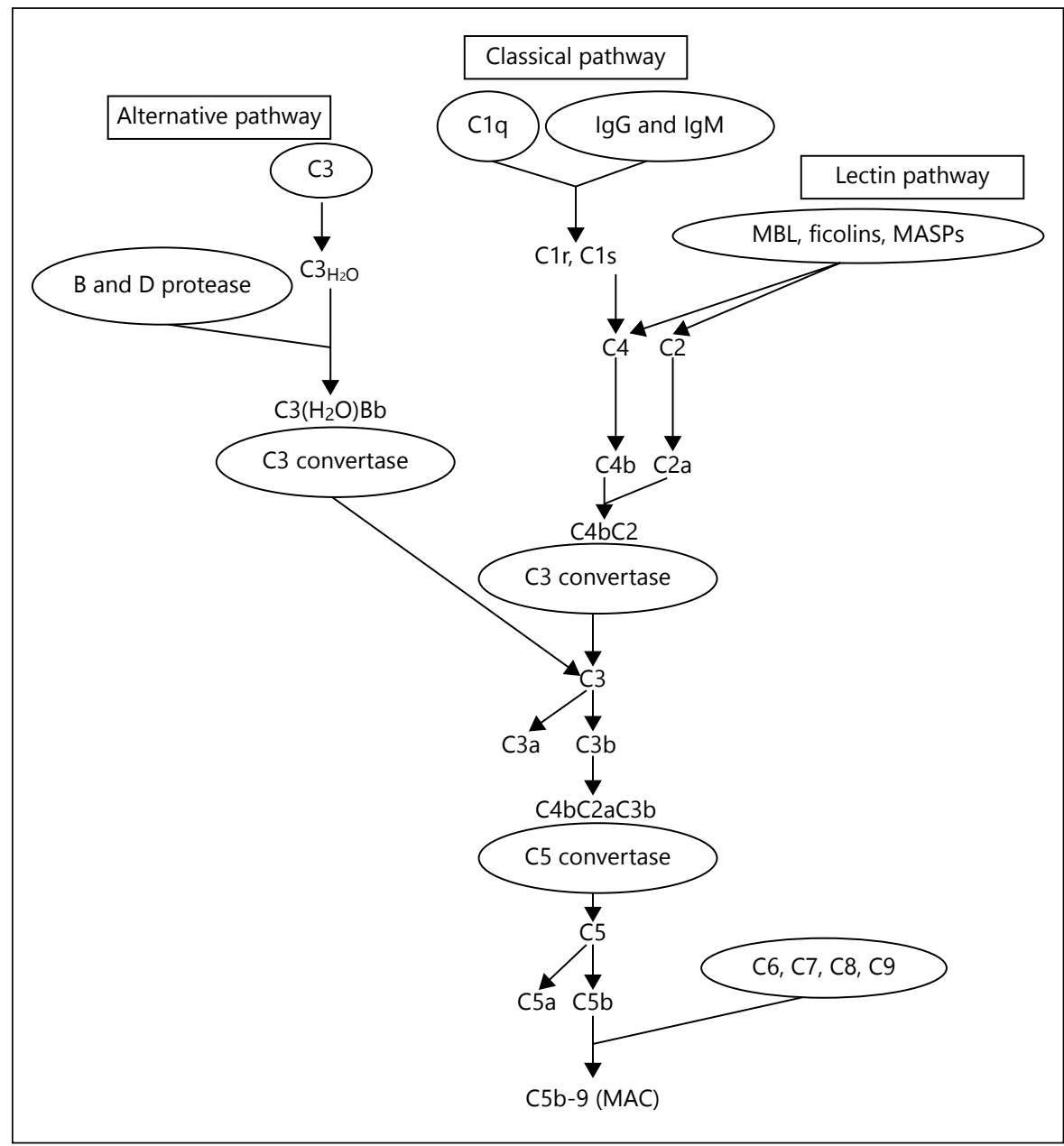

accomplish the chain reaction from $\mathrm{C} 5$ to $\mathrm{C} 9$. The factor $B$ and D protease can bind the hydrolyzate $\mathrm{C} 3\left(\mathrm{H}_{2} \mathrm{O}\right)$, which is a hydrolyzing $\mathrm{C} 3$ molecule. This binding generates $\mathrm{C} 3$ convertase $\mathrm{C} 3\left(\mathrm{H}_{2} \mathrm{O}\right) \mathrm{Bb}$ that cleave $\mathrm{C} 3$ into $\mathrm{C} 3 \mathrm{a}$ and $\mathrm{C} 3 \mathrm{~b}$ fragments $[22,24]$. The $\mathrm{C} 3 \mathrm{~b}$ induces formation of AP C 5 convertase. The convertase cleaves C5 into anaphylatoxin $\mathrm{C} 5 \mathrm{a}$ and fragments $\mathrm{C} 5 \mathrm{~b}$. Finally, the $\mathrm{C} 5 \mathrm{~b}$ plays an important role in the cascade $(\mathrm{C} 6, \mathrm{C} 7, \mathrm{C} 8, \mathrm{C} 9)[19,25]$.

\section{Mast Cell Activation-Related Pseudoallergy}

In multifarious pathological and physiological processes, mast cells that release differential mediators play very important roles. In non-IgE-mediated anaphylactoid reactions, various components (IgG, complement components, neuropeptides, cytokines, chemokines, and other inflammatory products) [26-30] directly stimulate the degranulation of mast cells and the selective release of mediators. Besides these endogenous stimuli, some exogenous molecules, which cause drug side effects can also directly activate mast cells [31], such as a vitamin $K_{1}$ injection or a Qingkailing injection [32, 33]. Different from IgE-mediated allergy, which is mediated by cell-surface receptors, the drugs are able to induce mast cell degranulation and alter enzymatic activity through various pathways $[34,35]$. Cysteinyl LTs (cysLTs) and histamine, released by mast cells, mediate bronchoconstriction [36].

\section{Stimulation via Exogenous Molecules}

Mast cells can be activated directly by many small molecular weight drugs. The activation is often associated with systemic pseudoallergenic and peptidergic drugs $[29,34]$. It has been reported that some exogenous molecules, such as compound $48 / 80$, can stimulate phospholipase D activity. Phospholipase D gives rise to the generation of endogenous phospholipids, which activate the lysophosphatidic acid receptor [32]. This property con- 
Table 1. Drugs causing pseudoallergy

\begin{tabular}{|c|c|c|c|c|c|}
\hline Opioid & Liposomal & Micelle-solubilized & NSAID & TCMIs & Other \\
\hline Morphine & Abelcet & Cyclosporine & Aspirin & Shuanghuanglian injection & $\begin{array}{l}\text { Vitamin } \mathrm{K}_{1} \\
\text { injection }\end{array}$ \\
\hline Codeine & Ambisome & Elitec & Dolobid & $\begin{array}{l}\text { Potassium dehydroandrogropolide } \\
\text { succinate injection }\end{array}$ & Rocuronium \\
\hline Hydromorphon & Doxil & Taxol & Voltaren & Xuesaitong injection & \\
\hline Oxycodone & Caelyx & Taxotere & Motrin & Danshen injection & \\
\hline Methadone & Myocet & Vumon & Naprosyn & Andrographis injection & \\
\hline
\end{tabular}

Buprenorphine

tributes to $\mathrm{G}$ protein-activation. The activated $\mathrm{G}$ proteins can produce exocytosis from mast cells, the synthesis of phosphatidylinositol 3-kinase, and the formation of arachidonate metabolites $[31,37]$. The stimulus of phosphatidylinositol 3-kinase activates phospholipase C gamma, which in turn invokes the hydrolysis of phosphatidylinositol 4,5-bisphosphate generating inositol 1,4,5-trisphosphate and diacylglycero. These mediators induce an increase in the level of intracellular $\mathrm{Ca}^{2+}$, which in turn activates protein kinase $\mathrm{C}$ [35]. This series of reactions causes mast cell degranulation and the release of inflammatory mediators generated by the PTK-PLA2 pathway [31].

\section{Stimulation via Complement Factors}

Complement is activated and anaphylatoxins are formed during pseudoallergy. C3a and C5a can induce respiratory distress, which results from the constriction of smooth muscle in the bronchioles and pulmonary arteries, and aggregation of leukocytes and platelets in the pulmonary blood vessels. C3a and C5a produce their effects by binding to specific $\mathrm{C} 3 \mathrm{a}$ receptor $(\mathrm{C} 3 \mathrm{aR})$ and $\mathrm{C} 5 \mathrm{a}$ receptor $(\mathrm{C} 5 \mathrm{aR})$, respectively. $\mathrm{C} 3 \mathrm{aR}$ and $\mathrm{C} 5 \mathrm{aR}$ proteins and mRNA are expressed by bronchial smooth muscle and epithelial cells in both mouse and human lung [38]. Moreover, mast cells express $\mathrm{C} 3 \mathrm{aR}$ and $\mathrm{C} 5 \mathrm{aR}$, which are 7 transmembrane domain $\mathrm{G}$ protein-coupled receptors [27]. C3a and C5a are mediated via the activation of their respective $G$ protein-coupled receptors in mast cells $[27,39,40]$. It has been reported that C3a activates mast cells either via the activation of mast cell surface $G$ protein-coupled receptors when the C3a level is low, or via the direct activation of $G$ proteins when the level is high $[27,41]$. It has been demonstrated that C3a, which can give rise to extracellular signal regulated kinase and AKT phosphorylation, causes a robust degranulation and activates different signaling pathways to induce chemokine production in human mast cells [42]. The anaphylatoxins $\mathrm{C} 3 \mathrm{a}$ and $\mathrm{C} 5 \mathrm{a}$ have high activity in stimulating chemotaxis and mobilization of intracellular free $\mathrm{Ca}^{2+}$ in mast cells [43]. C5a causes the rapid release of histamine and tryptase from mast cells, which can be activated in vitro by therapeutic and diagnostic agents [44].

\section{Drug-Induced Pseudoallergy}

It should be mentioned that some agents (Table 1), such as liposomal drugs [14], vitamin $\mathrm{K}_{1}$ injection [32] and TCMI, can induce pseudoallergy, though some investigators believe that the reactogenicity of liposomal drugs is overlooked, as they are not standard entries in approved lists of pseudoallergenic drugs [14]. It has been confirmed that the reactions caused by liposomal drugs are not IgE-mediated but rather via CARPA.

\section{Opioid Drugs}

Opioid medications are typically used to treat fractureassociated pain but have well-known adverse effects [45]. Nearly all opioids can cause pseudoallergy by a mechanism that directly triggers mast cell degranulation [46, 
47]. It has been reported that opioid drugs, such as codeine, meperidine and morphine can induce pseudoallergy [48]. Opioids induce histamine release by directly activating mast cells and cause flushing or pruritus that is nearly always mislabeled as allergy symptoms [49]. It has been found that codeine and morphine are more prone to inducing mast cell degranulation than other opioids [50]. Codeine induces pseudoallergy via a non-immunologic mechanism, which is independent of both IgE and the high-affinity IgE receptor FceRI [51, 52]. The activation of mast cell degranulation by codeine may, therefore, occur through its actions at the opioid receptor [51]. There are 3 recognized types of opioid receptors, namely, mu $(\mu)$, delta $(\delta)$, and kappa $(\kappa)[53,54]$. It has been demonstrated that human mast cells express mRNA for $\delta$ - and $\mu$-receptors but not for $\kappa$-opioid receptors [51]. Human cutaneous mast cells possess opioid receptors, which when stimulated trigger degranulation. These receptors are activated by the $\mu$-receptor agonists - morphine, codeine and meperidine [55]. There are 2 known pathways that stimulate the degranulation of mast cells: direct $G$ protein-activation and activation through opioid receptor stimulation [56]. Activation of these receptors triggers intracellular signaling pathways mediated by $G$ proteins, ion channel modulation and mitogen-activated protein kinase activation [51]. Codeine preferentially activates the $\mu$-receptor, but the synthetic analog of codeine, meperidine, does not activate human mast cell mediator production at the same doses [51]. The morphine-induced wheal-and-flare response is caused by the activation of a naloxone sensitive $\mu$-opioid receptor on mast cells [55]. Opioid drug-induced stimulation of mast cells is associated with phospholipase $\mathrm{C}$ activity attributed to opioid receptor activation $[48,57]$.

\section{Complement Activation-Related}

Pseudoallergenic Drugs: Liposomal Drugs and

Micelle-Solubilized Drugs

CARPA can be a severe syndrome involving several cardio pulmonary symptoms that include pulmonary edema, hypotension, arrhythmia, airway occlusion, respiratory distress, and potential cardiac arrest, which may eventually lead to anaphylactic cardiogenic shock and death in severe cases [16]. Intravenous injection of nanotechnology drugs (liposomal, micellar) may induce CARPA [14]. Liposomal drugs can also activate CARPA. Liposome activation of AP occurs through mechanisms independent of antibodies through direct $\mathrm{C} 3$ adsorption and altered conformational changes that resemble $\mathrm{C} 3 \mathrm{~b}$, ultimately triggering the production of $\mathrm{C} 3 \mathrm{Bb}$ convertase
[58]. The CP activated by liposomes involves 3 mechanisms: (1) antibodies (IgG and Immunoglobulin M) attaches to the phospholipid head-groups and cholesterol attaches to vesicles; (2) direct Clq binds to liposomes with high-density anionic charges derived from phospholipids; and (3) liposomal activation of the CP via $\mathrm{C}$-reactive protein binding occurs, which activates the complement system by acting on the globular head of $\mathrm{Clq}$ [58]. Liposome-mediated activation of LP has been rarely reported. MBL binding to phosphatidylinositol-incorporated liposomes presumably induces MASP-2 activation and the triggering of the complement system [58]. The most well-known liposomal drugs are ambisome and doxil, and the micelle-solubilized-drug taxol [14]. Ambisome, a charged non-PEGylated liposome, induces CARPA via AP $[16,59]$. Factor $H$ inhibits complement activation induced by the antifungal drugs ambisome and taxol [16]. Doxil, one of the PEG-PL-engineered nanomedicines, triggers the complement activation in human serum through both CP and AP [21]. In addition, PEG may act directly on complement proteins and suppress protein adsorption [21].

\section{Non-steroidal Anti-Inflammatory Drugs}

NSAIDs alleviate inflammation (swelling) and pain (analgesia) by inhibiting different isoforms of cyclooxygenases (COXs) and by reducing the synthesis of prostaglandins. COX-1 plays an important role in mucosal protection and physiological homeostasis [48]. It has been shown that the adverse effects of NSAID are induced by COX-1 inhibition [60]. Aspirin and single-dose NSAIDs induce pseudoallergy, but some NSAIDs are well tolerated [3]. Unexpectedly, the use of acetic and propionic derivatives has a relatively high risk for developing anaphylactic reactions [61]. For instance, aspirin inhibits COXs by converting arachidonic acid to various prostanoids, resulting in the synthesis of LTs and a decreased synthesis of prostaglandin $\mathrm{E}_{2}[47,48]$. NSAIDs inhibit the function of COX, which directs the metabolism of arachidonic acid towards the 5-lipoxygenase pathway, resulting in an increase of cysLT synthesis [62]. Two types of LTs are formed by the 5-lipoxygenase-mediated oxidation of arachidonic acid into the unstable intermediate, $\mathrm{LTA}_{4}$, namely, cysLT and $\mathrm{LTB}_{4}$. CysLTs are generated in mast cells and basophils when $\mathrm{LTA}_{4}$ is conjugated to form reduced glutathione by $\mathrm{LTC}_{4}$ synthase. The cysLT, $\mathrm{LTC}_{4}$, is extracellularly converted into $\mathrm{LTD}_{4}$ and then to cysLTs, a stable metabolite, which acts on $\mathrm{G}$ protein-coupled $\mathrm{LTE}_{4}$ receptors to induce bronchoconstriction, vascular leakage and eosinophilia $[63,64]$. 
Traditional Chinese Medicine Injections

Due to high bioavailability, TCMIs are widely used for treatment. However, pseudoallergy is frequently induced by TCMs. Previous studies revealed that TCMIs can directly induce $\beta$-hexosaminidase and histamine release through mast cell degranulation [65]. Due to the complexity of the TCMI components, it is important to screen and identify allergenic constituents contained in TCMIs. It has been reported that Tween-80, known as a dispersing agent and solubilizer in TCMI, induces pseudoallergy in zebrafish [66]. Here, the Xuesaitong injection is employed as a typical example of TCMIs. Mediators such as histamine and $\beta$-hexosaminidase released from mast cells and RBL-2H3 cells are stimulated by Xuesaitong injection [67]. Xuesaitong-induced pseudoallergy occurs via direct stimulation, the complement and the kallikreinkinin pathways [68]. In addition, substances that induce an anaphylactoid effect induce histamine, LTB4, uric acid and other drugs, which have been confirmed to be involved in arginine and proline metabolism [68].

\section{Other Drugs}

In addition, it has been shown that vitamin $K_{1}$ injections induce pseudoallergy but not anaphylaxis [32]. However, vitamin $\mathrm{K}_{1}$ itself does not induce pseudoallergy; the trigger may be the solubilizer. Perioperatively administered rocuronium can induce pseudoallergy via the nonIgE mediated Mas-related $G$ protein-coupled receptor member X2, which activates mast cell degranulation [69].

\section{Conclusions}

Pseudoallergy is mediated through IgE-independent mechanisms, which has received much research attention of late. This review has shown that many drugs widely used in the clinic can cause severe, occasionally lethal, pseudoallergy, which still remains an unsolved public health issue. Advances in this field will ensure the safety of new drugs in terms of preventing adverse reactions. Two main mechanisms are summarized in considerable detail, providing guidelines for the ultimate eradication of pseudoallergy. The screening method of components in medications should be further improved. More discussion and research in this field will contribute to the development of effective diagnosis methods in the clinic.

\section{Acknowledgement}

We specially thank Xinyue Zhang for the manuscript modification. The present manuscript was supported by grants from the Natural Science Foundation of Zhejiang Province (Y15H280032). The authors are grateful to all study participants.

\section{Disclosure Statement}

The authors declare that they have no competing interests.

\section{References}

1 Szebeni J: Complement activation-related pseudoallergy: a new class of drug-induced acute immune toxicity. Toxicology 2005;216: 106-121.

2 Waller DG: Allergy, pseudo-allergy and nonallergy. Br J Clin Pharmacol 2011;71:637-638.

3 Drug allergy: an updated practice parameter. Ann Allergy Asthma Immunol 2010;10:259273.

4 Qiu S, et al: Complement activation associated with polysorbate 80 in beagle dogs. Int Immunopharmacol 2013;15:144-149.

$5 \mathrm{He} \mathrm{SH}$, et al: Mast cells and basophils are essential for allergies: mechanisms of allergic inflammation and a proposed procedure for diagnosis. Acta Pharmacol Sin 2013;34:1270-1283.

6 Jurakić Toncić R, et al: Nonallergic hypersensitivity to nonsteroidal antiinflammatory drugs, angiotensin-converting enzyme inhibitors, radiocontrast media, local anesthetics, volume substitutes and medications used in general anesthesia. Acta Dermatovenerol Croat 2009;17:54-69.
7 Jurakić Toncić R, Marinović B, Lipozencić J: Nonallergic hypersensitivity to nonsteroidal antiinflammatory drugs, angiotensin-converting enzyme inhibitors, radiocontrast media, local anesthetics, volume substitutes and medications used in general anesthesia. Acta Dermatovenerol Croat 2009;17:54-69.

8 Wang Z, et al: Experimental study on anaphylaxis of qingkailing injection and its components on Beagle dogs. J Tradit Chin Med 2012; 32:641-645.

9 Demoly P, et al: Predictive capacity of histamine release for the diagnosis of drug allergy. Allergy 1999;54:500-506.

10 Parratt JR, West GB: 5-Hydroxytryptamine and the anaphylactoid reaction in the rat. J Physiol 1957;139:27-41

11 Ehlers I, et al: Role of nonallergic hypersensitivity reactions in children with chronic urticaria. Allergy 1998;53:1074-1077.

12 Ring J, Messmer K: Incidence and severity of anaphylactoid reactions to colloid volume substitutes. Lancet 1977;1:466-469.
13 Szebeni J, et al: Formation of complementactivating particles in aqueous solutions of Taxol: possible role in hypersensitivity reactions. Int Immunopharmacol 2001;1:721735 .

14 Szebeni J: Complement activation-related pseudoallergy: a stress reaction in blood triggered by nanomedicines and biologicals. Mol Immunol 2014;61:163-173.

15 Sercombe L, et al: Advances and challenges of liposome assisted drug delivery. Front Pharmacol 2015;6:286.

16 Mészáros T, et al: Factor $\mathrm{H}$ inhibits complement activation induced by liposomal and micellar drugs and the therapeutic antibody rituximab in vitro. Nanomedicine 2016;12: 1023-1031.

17 Jeon B, et al: In vitro and in vivo downregulation of $\mathrm{C} 3$ by lipoteichoic acid isolated from Lactobacillus plantarum K8 suppressed cytokine-mediated complement system activation. FEMS Microbiol Lett 2016; 363:pii:fnw140. 
18 Barnes MG, Weiss AA: BrkA protein of Bordetella pertussis inhibits the classical pathway of complement after $\mathrm{C} 1$ deposition. Infect Immun 2001;69:3067-3072.

19 Mella A, et al: Complement cascade and kidney transplantation: the rediscovery of an ancient enemy. World J Transplant 2014;4:168175.

20 Ehrnthaller C, et al: New insights of an old defense system: structure, function, and clinical relevance of the complement system. Mol Med 2011;17:317-329.

21 Moghimi SM, et al: Complement activation cascade triggered by PEG-PL engineered nanomedicines and carbon nanotubes: the challenges ahead. J Control Release 2010;146: 175-181.

22 Ricklin D, et al: Complement: a key system for immune surveillance and homeostasis. Nat Immunol 2010;11:785-797.

23 Gaboriaud C, et al: Structure and activation of the $\mathrm{C} 1$ complex of complement: unraveling the puzzle. Trends Immunol 2004;25:368-373.

24 Mella A, et al: Complement cascade and kidney transplantation: the rediscovery of an ancient enemy. World J Transplant 2014;4:168-175.

25 Smith RJ, Harris CL, Pickering MC: Dense deposit disease. Mol Immunol 2011;48:16041610.

26 Woolhiser MR, et al: IgG-dependent activation of human mast cells following up-regulation of FcgammaRI by IFN-gamma. Eur J Immunol 2001;31:3298-3307.

27 Ali H: Regulation of human mast cell and basophil function by anaphylatoxins $\mathrm{C} 3 \mathrm{a}$ and C5a. Immunol Lett 2010;128:36-45.

28 Alysandratos KD, et al: Neurotensin and $\mathrm{CRH}$ interactions augment human mast cell activation. PLoS One 2012; 7:e48934.

29 Bischoff SC: Role of mast cells in allergic and non-allergic immune responses: comparison of human and murine data. Nat Rev Immunol 2007;7:93-104.

30 Juremalm M, Nilsson G: Chemokine receptor expression by mast cells. Chem Immunol Allergy 2005;87:130-144.

$31 \mathrm{Yu}$ Y, et al: Non-IgE mediated mast cell activation. Eur J Pharmacol 2016;778:33-43.

$32 \mathrm{Mi} \mathrm{YN}$, et al: The severe adverse reaction to vitamin $\mathrm{K} 1$ injection is anaphylactoid reaction but not anaphylaxis. PLoS One 2014; 9:e90199.

33 Yi Y, et al: [Evaluation and study of pseudoallergic reactions of three kinds of traditional Chinese medicine injections and its mechanisms]. Zhongguo Zhong Yao Za Zhi 2015; 40:2711-2716.

34 Ferry X, et al: G protein-dependent activation of mast cell by peptides and basic secretagogues. Peptides 2002;23:1507-1515.

35 Palomäki VA, Laitinen JT: The basic secretagogue compound $48 / 80$ activates G proteins indirectly via stimulation of phospholipase D-lysophosphatidic acid receptor axis and 5-HT1A receptors in rat brain sections. Br J Pharmacol 2006;147: 596-606.
36 Nomani S, Cockcroft DW, Davis BE: Allergen inhalation challenge, refractoriness and the effects of ibuprofen. Allergy Asthma Clin Immunol 2016;12:24.

37 Moser E, et al: G protein-coupled receptorassociated sorting protein 1 regulates the postendocytic sorting of seven-transmembrane-spanning $\mathrm{G}$ protein-coupled receptors. Pharmacology 2010;86:22-29.

38 Drouin SM, et al: Expression of the complement anaphylatoxin C3a and C5a receptors on bronchial epithelial and smooth muscle cells in models of sepsis and asthma. J Immunol 2001;166:2025-2032.

39 Ahamed J, Ali H: Distinct roles of receptor phosphorylation, $G$ protein usage, and mitogen-activated protein kinase activation on platelet activating factor-induced leukotriene $\mathrm{C}(4)$ generation and chemokine production. J Biol Chem 2002;277:2268522691.

40 Ali $\mathrm{H}$, et al: Differences in phosphorylation of formylpeptide and C5a chemoattractant receptors correlate with differences in desensitization. J Biol Chem 1993;268:2424724254.

41 Mousli M, et al: Peptidergic pathway in human skin and rat peritoneal mast cell activation. Immunopharmacology 1994;27:111.

42 Venkatesha RT, et al: Distinct regulation of C3a-induced MCP-1/CCL2 and RANTES/ CCL5 production in human mast cells by extracellular signal regulated kinase and PI3 kinase. Mol Immunol 2005;42:581587.

43 Hartmann K, et al: C3a and C5a stimulate chemotaxis of human mast cells. Blood 1997; 89:2863-2870.

44 Genovese A, et al: Human cardiac mast cells in anaphylaxis. Chem Immunol Allergy 2010; 95:98-109.

45 Baker MD, Gullett JP: Ultrasound-guided femoral nerve blocks. Pediatr Emerg Care 2015;31:864-868; quiz 869-871.

46 Trescot AM: Opioid Pharmacology and Pharmacokinetics, 2016, pp 45-62.

47 Becker DE: Drug allergies and implications for dental practice. Anesth Prog 2013;60:188 197.

48 Wang $\mathrm{H}$, et al: Agents that induce pseudo-allergic reaction. Drug Discov Ther 2011;5: 211-219.

49 Stollery N: Allergic reactions. Practitioner 2015;259:34-35.

50 Tahmasebi A, Skytén J: An in vitro Study of Drug-Induced Degranulation of Human HMC-1 Mast Cells and Rat RBL-2H3 Cells, 2015.

51 Sheen $\mathrm{CH}$, Schleimer RP, Kulka M: Codeine induces human mast cell chemokine and cytokine production: involvement of G-protein activation. Allergy 2007;62: 532-538.

52 Yoo HS, et al: A case of codeine induced anaphylaxis via oral route. Allergy Asthma Immunol Res 2014;6:95-97.
53 Chen ZC, et al: Activation of peripheral opioid $\mu$-receptors in blood vessel may lower blood pressure in spontaneously hypertensive rats. Pharmacology 2011;87:257-264.

54 Minami K, et al: Sevoflurane inhibits the $\mu$-opioid receptor function expressed in xenopus oocytes. Pharmacology 2011;88:127132.

55 Casale TB, et al: Induction of human cutaneous mast cell degranulation by opiates and endogenous opioid peptides: evidence for opiate and nonopiate receptor participation. J Allergy Clin Immunol 1984;73:775781.

56 Tahmasebi A, Skytén J: An in vitro study of drug-induced degranulation of human HMC1 mast cells and rat RBL-2H3 cells. Umeå Universitet 2014, pp 9-11.

57 Liu NJ, vonGizycki H, Gintzler AR: Phospholipase Cbeta1 modulates pain sensitivity, opioid antinociception and opioid tolerance formation. Brain Res 2006;1069:47-53.

58 Logothetidis S (ed): Nanomedicine and Nanobiotechnology. Berlin, Springer, 2014.

59 Dézsi L, et al: Features of complement activation-related pseudoallergy to liposomes with different surface charge and PEGylation: comparison of the porcine and rat responses. J Control Release 2014;195:2-10.

60 Dannhardt G, Kiefer W: Cyclooxygenase inhibitors - current status and future prospects. Eur J Med Chem 2001;36:109-126.

61 Quiralte J, et al: Challenge-based clinical patterns of 223 Spanish patients with nonsteroidal anti-inflammatory-drug-induced-reactions. J Investig Allergol Clin Immunol 2007; 17:182-188.

62 Wang H, Wang HS, Liu ZP: Agents that induce pseudo-allergic reaction. Drug Discov Ther 2011;5:211-219.

63 Laidlaw TM, et al: Prostaglandin E2 resistance in granulocytes from patients with aspirinexacerbated respiratory disease. J Allergy Clin Immunol 2014;133:1692-1701.e3.

64 Singh RK, et al: Cysteinyl leukotrienes and their receptors: molecular and functional characteristics. Pharmacology 2010;85:336-349.

65 Han S, et al: New method of screening allergenic components from shuanghuanglian injection: with RBL-2H3/CMC model online HPLC/MS system. J Pharm Biomed Anal 2014;88:602-608.

66 Yang R, et al: Tween-80 and impurity induce anaphylactoid reaction in zebrafish. J Appl Toxicol 2015;35:295-301.

67 Xiang Z, et al: The anaphylactoid constituents in Xue-Sai-Tong injection. Planta Med 2013; 79:1043-1050.

$68 \mathrm{Xu} \mathrm{Y}$, et al: Integrative analysis of proteomics and metabolomics of anaphylactoid reaction induced by Xuesaitong injection. J Chromatogr A 2015;1416:103-111.

69 Spoerl D, et al: Non-IgE-dependent hypersensitivity to rocuronium reversed by sugammadex: report of three cases and hypothesis on the underlying mechanism. Int Arch Allergy Immunol 2016;169:256-262. 